\title{
Extensive linkage disequilibrium in a wild bird population
}

\author{
MH Li and J Merilä \\ Ecological Genetics Research Unit, Department of Biological and Environmental Sciences, University of Helsinki, Helsinki, Finland
}

Knowledge about the extent and patterns of linkage disequilibrium (LD) can provide important insights into demographic processes and strategies to identify the genetic basis of complex phenotypes in wild populations. However, data on the extent and patterns of LD from non-model vertebrate species from the wild are still scarce. We conducted so far the most extensive and detailed examination of LD in a pedigreed wild bird population using genotypes from 97 autosomal and 6 gonosomal microsatellites and a recently established linkage map of Siberian jays (Perisoreus infaustus). Analysis of syntenic marker pairs showed high levels of LD that extended over tens of centimorgans or several megabases and generally decayed as an increasing function of intermarker distance. In addition, significant LD was also very common between nonsyntenic markers. Patterns of LD varied across different linkage groups possibly because of the differences in chromosomal structure (macro-, micro-, and Z-chromosome). In particular, the level of LD was significantly lower on the Z-chromosome than on the autosomes at comparable genetic distances. In general, the high levels and extent of LD in this population are likely owing to its relatively small size, significant intrapopulation genetic structure, and occurrence of inbreeding. Whatever the cause, the long-range LD between syntenic loci suggests that LD mapping of phenotypic traits in this population using low-density markers maps is feasible. However, the frequent occurrence of LD between nonsyntenic markers suggests that the combined use of linkage and LD methods is needed to reduce the likelihood of false-positive associations between marker loci and traits of ecological and evolutionary interest.

Heredity (2010) 104, 600-610; doi:10.1038/hdy.2009.150; published online 4 November 2009

Keywords: genome wide; linkage disequilibrium; Siberian jay; microsatellites

\section{Introduction}

The levels and extent of linkage disequilibrium (LD) are affected by many factors, including population size and structure, mating pattern, selection, genetic drift, migration, admixture, mutation, gene conversion, epistasis, and recombination (Ardlie et al., 2002). Analysis of LD can thus give insights into demographic history and dynamics of a given population. It has been shown to be a very powerful approach to distinguish between alternative models (for example, the hitchhiking vs the background selection models) of genome evolution (Lewontin, 1964). In addition, information about the patterns of LD can be used for mapping genes or quantitative trait loci (QTL), particularly in species where traditional pedigree-based QTL-mapping approaches are not feasible as is the case with many wild species (see Rafalski and Morgante, 2004).

There has been a number of investigations about the levels and extent of LD in humans (for example, Stephens et al., 2001), domesticated animals (for example, Farnir et al., 2000), plants (for example, Remington et al., 2001), and model organisms such as Drosophila (for example, Nordborg et al., 2002). In addition, LD has also

Correspondence: $\operatorname{Dr}$ MH Li, Ecological Genetics Research Unit, Department of Biological and Environmental Sciences, University of Helsinki, PO Box 65, Helsinki 00014, Finland.

E-mail: menghua.li@helsinki.fi

Received 6 May 2009; revised 21 September 2009; accepted 25 September 2009; published online 4 November 2009 been used successfully and extensively to investigate the evolutionary history of certain bacteria of zoonotic importance (for example, Wilson et al., 2008). However, detailed studies to the effect of LD from wild vertebrate populations are confined to a few studies of birds (Edwards and Dillon, 2004; Backström et al., 2006; Balakrishnan and Edwards, 2009) and mammals (McRae et al., 2005; Laurie et al., 2007; Slate and Pemberton, 2007). However, many key questions in evolutionary biology and genetics cannot be addressed using model systems or domesticated populations (for example, Gracey and Cossins, 2003), and studies of wild populations can provide important insights into evolutionary genetics not traceable in the laboratory or captivity (for example, McRae et al., 2005; Ellegren and Sheldon, 2008). Thus, the measures of LD may well find their application in future studies of wild populations, in which there is considerable current interest in linkage and QTL mapping and the subsequent study of the genetics of traits of ecological importance (for example, Backström et al., 2006; Gratten et al., 2008; Stapley et al., 2008). However, studies of LD in wild populations made so far are characterized by a limited number of analyzed chromosomes/linkage groups (LGs). However, the information on the structure of LD at the genome level is crucial for determining appropriate experimental design and guiding the selection and spacing of markers to create marker maps useful in candidate gene, candidate region, and eventually whole-genome association studies (De La Vega et al., 2005). 
The overall extent of LD across the genome is one of several critical attributes, which affects how readily phenotypic traits in natural populations can be mapped using whole-genome association studies (Flint-Garcia et al., 2003). On the one hand, extensive LD allows associations to be detected more readily using a small number of distantly placed markers, whereas low LD necessitates fine-scale mapping. On the other hand, long extending LD is a potential barrier to the localization of causative polymorphisms of phenotypic effects, whereas gene identification might be less tedious in the case of limited LD. Data from humans, livestock, and selfing plants have shown a general pattern of decaying LD with physical/recombination distance between markers. However, the rate of this decay varies among species, populations, and different parts of the genome (see Kolkman et al., 2007; Slate and Pemberton, 2007).

The aim of this study was to investigate the magnitude and extent of LD in the genome of a wild population of Siberian jays (Perisoreus infaustus) making use of 103 microsatellite markers genotyped in 349 individuals of 5 major families. We examined and compared the strength of pairwise LD between syntenic and nonsyntenic markers using three different measures of strength of association. In particular, we were interested in learning how far-in terms of centimorgans-the significant linkage in the Siberian jay genome extends. By extrapolating the LD measured on the genome scale, this study aimed to explore the prospects and strategies for LD-based association mapping in a wild bird population.

\section{Materials and methods}

Study species, study population, and pedigree

The Siberian jay ( $P$. infaustus) is a relatively long-lived (average generation time ca. 4 years) oscine passerine bird from the Corvidae family. It has a stable socially monogamous breeding system in which lifelong pair bonds are formed in permanent territories established in the coniferous forest of the northern Eurasian taiga (Ekman et al., 1994). Siberian jays live in small territorial groups (3-5 individuals) consisting of a breeding (alpha) pair, their retained offspring, and/or unrelated immigrants (Ekman et al., 1994). The species is also highly sedentary and philopatric so that closely related males often hold neighboring territories (Ekman et al., 2001).

A long-term field study of the Siberian jay (P. infaustus) population in Suupohja (ca. $66^{\circ} 18^{\prime} \mathrm{N}, 29^{\circ} 29^{\prime} \mathrm{E}$ ) in Western Finland has been conducted since 1974. The study population, field methods and the data structure are described in detail by Lillandt et al. (2001, 2003), Alho et al. (2009) and, in particular, Jaari et al. (2009). The study population is in a semi-isolated forest area near the southwestern border of the species' breeding range in Finland, where its population density is much lower than within the continuous main distribution area in the north (Kemppainen, 2002). Siberian jays were monitored mainly in three neighboring forest areas, separated from each other by $100-1500 \mathrm{~m}$ wide agricultural fields or peatlands.

The pedigree, hereafter the Suupohja pedigree, used in this study was built based on field observations (Lillandt et al., 2001) and molecular tools as described in Jaari et al.
(2009). The pedigree is composed of five major families consisting of 349 animals and was from a large Siberian jay data set comprising more than 1000 individuals sampled in 1975-2006. The five family pedigrees varied in depth from four to six generations and ranged in size from 65 to 73 animals. These family pedigree configurations yield large numbers of pairwise relationships, which include, but are not limited to, parentoffspring, full-sib, half-sib, grandparent-grandchild, great grandparent-great grandchild, avuncular, halfavuncular, and first cousin relationships. A more thorough description of the structure of the mapping pedigree can be found from Lillandt et al. (2001, 2003) and Jaari et al. (2009).

As the levels and extent of LD are expected to be higher among closely related individuals than among more distantly related individuals (for example, Slate and Pemberton, 2007), we also analyzed LD in a restricted data consisting on the founders of pedigree data. To this effect we confined our analyses (see below) to those 98 individuals that were assumed to be unrelated in the first two or three generations in the five family pedigrees. We reasoned that if the patterns and extent of LD are roughly similar between analyses based on pedigree and founder data, then the results of the pedigree analyses can be generalized to the whole population data.

\section{Microsatellite markers and genotypes}

The pedigree has been genotyped in a total of 107 microsatellites comprising 101 autosomal and $6 \mathrm{Z}$-chromosomal markers (Jaari et al., 2009). A genetic map has been constructed by following the co-segregation within the pedigree and determining marker order and distances with the software CRIMAP (Green et al., 1990), described in detail in Jaari et al. (2009). The microsatellites were distributed in nine autosomal LGs (LG1-LG9) and one Z-chromosome LG (LGZ) covering $886.6 \mathrm{cM}$ in total. Eight LGs (LGs 1-7 and Z) that contain three or more markers (3-29) were analyzed here, totalling 103 microsatellite markers. The length of LGs varied from 26.4 to $185.6 \mathrm{cM}$ with the average intermarker distance ranging from $4.42 \mathrm{cM}$ on LGZ to $17.02 \mathrm{cM}$ on LG3 (Jaari et al., 2009). The marker order and all pairwise intermarker genetic distances are based on the sex-averaged linkage maps of Siberian jay (see: http://www.biomedcentral.com/content/pdf/1471-216410-1.pdf).

\section{Microsatellite variation and between-family genetic structure}

Microsatellite variation was assessed by estimating the number of alleles and the observed heterozygosity using the Excel Microsatellite Toolkit version 3.1.1 (Park, 2001). Within the population, genetic differentiation among families was quantified by the $\theta$ estimator of $F_{\mathrm{ST}}$ (Weir and Cockerham, 1984). We also calculated the value of $f$, which corresponds to Wright's (1951) within-population inbreeding coefficient $F_{\mathrm{IS}}$, across the loci over the total samples. These calculations were performed with FSTAT version 2.9.3.2 (Goudet, 2002) and the significance and the $95 \%$ confidence intervals of $\theta$ and $f$ were determined by 10000 permutations. 
Measures of LD and statistical significance of association The strength of LD was measured using two different metrics. First, we used Lewontin's normalized $D^{\prime}$ (Lewontin, 1964) modified for multiple alleles (Hedrick, 1987). This statistic has been used earlier to measure the extent of LD between markers spanning one single chromosome and/or the whole genome in humans (for example, Mohlke et al., 2001; Ardlie et al., 2002), livestock (for example, Farnir et al., 2000; Khatkar et al., 2006a, b), and wild vertebrates (for example, Backström et al., 2006; Slate and Pemberton, 2007). Apart from facilitating the comparison of the results with those other studies, $D^{\prime}$ is a convenient measure of LD as it allows use of highly polymorphic markers and is less sensitive to variation in marker allele frequencies and marker information content than other measures of LD (Hedrick, 1987; McRae et al., 2002). Following Hedrick (1987), LD between two multiallelic loci A and B was measured as

$$
D^{\prime}=\sum_{i=1}^{u} \sum_{v=1}^{v} p_{i} q_{j}\left|D_{i j}^{\prime}\right|
$$

where $u$ and $v$ are the respective number of alleles at the two loci, $p_{i}$ and $q_{i}$ are the population frequencies of marker allele $i$ at locus A and marker allele $j$ at locus B, and $\left|D_{i j}^{\prime}\right|$ is the absolute value of Lewontin's (1964) normalized LD measure computed as

$$
D_{i j}^{\prime}=\frac{D_{i j}}{D_{\max }}, \text { with } D_{i j}=p_{i j} p_{i} q_{j}
$$

where $p_{i j}$ is the observed frequency of gametes $A_{i} B_{j}$, and $p_{i}$ and $q_{j}$ are the frequencies of allele $A_{i}$ and $B_{j}$, respectively, and

$$
D_{\max }=\left[\begin{array}{l}
\min \left[p_{i} q_{j},\left(1-p_{i}\right)\left(1-q_{j}\right)\right] ; D_{i j}<0 \\
\min \left[p_{i}\left(1-q_{j}\right),\left(1-p_{i}\right) q_{j}\right] ; D_{i j}>0
\end{array}\right]
$$

Second, we used the standardized $\chi^{\prime 2}$ (Yamazaki, 1977; Hedrick, 1987). Simulation studies (for example, Zhao et al., 2005) have shown that $\chi^{\prime 2}$ is the preferred measure of LD for multiallelic markers for the purpose of QTL mapping, as it closely tracks the regression of allelic state at a QTL on allelic state at a multiallelic marker. Hence, it conveys the same information for multiallelic markers as $r^{2}$ (Hill and Robertson, 1968) for biallelic markers (Lipkin et al., 2009). The measure $r^{2}$ is useful for biallelic markers, as it stands in inverse proportion to the sample size needed to show significance. However, when pooling across allelic pairs for multiallelic markers, $r^{2}$ weighted by the product of allele frequencies was found to be strongly undervalued (Heifetz et al., 2005; Zhao et al., 2005). Hence, the measure $\chi^{\prime 2}$ is considered here instead of $r^{2}$, and $\chi^{\prime 2}$ was calculated as

$$
x^{\prime 2}=\frac{x^{2}}{N(n-1)}=\sum_{i=1}^{u} \sum_{j=1}^{v} \frac{D_{i j}^{2}}{p_{i} q_{j}(n-1)}
$$

where $N$ is the population size, and $n$ is the number of alleles at the marker with the smaller number of alleles, that is, $n=\min (u, v)$.

Multiallelic interallelic disequilibrium analysis software (MIDAS) (Gaunt et al., 2006) provided estimates of $D^{\prime}{ }_{i j}, p_{i}, q_{i}$, and $\chi^{2}$ for allelic combinations; $D^{\prime}$ and $\chi^{\prime 2}$ for each marker pair were then calculated using equations (1) and (4) above. The graphical visualization of interallelic disequilibrium between alleles of the multiallelic microsatellites was also provided by the software. It can offer insights into patterns of LD across genomic regions and inferences about patterns of evolution and selection. In addition, the program has been shown to considerably increase both the statistical power and the accuracy of estimation of the strength of LD (Gaunt et al., 2006).

In addition to MIDAS analyses, the statistical significance ( $P$-value) of the observed association between pairs of alleles under the null hypothesis of random allelic assortment was also estimated by a Monte-Carlo approximation of Fisher's exact test as implemented in the software Arlequin (Schneider et al., 2000) using a Markov chain extension to Fisher's exact test for $R \times C$ contingency tables (Slatkin, 1994). A total of 100000 alternative tables were explored by the Markov chain and probabilities ( $P$-values) were typically estimated with a standard error of $<0.001$. No attempt was made to correct for multiple testing, as the large number of tests would result in very low statistical power (see McRae et al., 2002).

The LD and $P$-values of significance were computed between all syntenic markers and between all pairs of nonsyntenic markers among the LGs from seven different data sets. Data set 1 comprised genotypes from the total Suupohja pedigree; data set 2 comprised genotypes from only the founders $(n=98$, Table 1$)$;

\begin{tabular}{|c|c|c|c|c|c|c|c|c|}
\hline \multirow[t]{2}{*}{ Data set } & \multirow[t]{2}{*}{$\mathrm{n}$} & \multirow[t]{2}{*}{ Metric } & \multicolumn{5}{|c|}{ Syntenic } & \multirow[t]{2}{*}{ Nonsyntenic } \\
\hline & & & $0-20 c M$ & $20-40 c M$ & $40-60 c M$ & $60-100 c M$ & $100-190 \mathrm{cM}$ & \\
\hline 1 (The pedigree) & 349 & $\begin{array}{l}D^{\prime} \\
\chi^{\prime 2} \\
P<0.05\end{array}$ & $\begin{array}{r}0.517(0.174) \\
0.264(0.098) \\
211 / 222(0.95)\end{array}$ & $\begin{array}{c}0.484(0.153) \\
0.187(0.074) \\
189 / 206(0.92)\end{array}$ & $\begin{array}{r}0.476(0.145) \\
0.174(0.072) \\
138 / 151(0.91)\end{array}$ & $\begin{array}{r}0.381(0.129) \\
0.168(0.067) \\
200 / 224(0.89)\end{array}$ & $\begin{array}{r}0.308(0.113) \\
0.160(0.058) \\
113 / 148(0.76)\end{array}$ & $\begin{array}{r}0.354(0.121) \\
0.165(0.061) \\
3567 / 4309(0.83)\end{array}$ \\
\hline 2 (Founders) & 98 & $\begin{array}{l}D^{\prime} \\
\chi^{\prime 2} \\
P<0.05\end{array}$ & $\begin{array}{r}0.483(0.144) \\
0.231(0.087) \\
204 / 222(0.92)\end{array}$ & $\begin{array}{r}0.462(0.151) \\
0.162(0.067) \\
162 / 206(0.79)\end{array}$ & $\begin{array}{r}0.456(0.137) \\
0.158(0.058) \\
104 / 151(0.69)\end{array}$ & $\begin{array}{r}0.364(0.119) \\
0.147(0.054) \\
117 / 224(0.52)\end{array}$ & $\begin{array}{c}0.282(0.101) \\
0.144(0.052) \\
61 / 148(0.41)\end{array}$ & $\begin{array}{r}0.362(0.127) \\
0.168(0.065) \\
2213 / 4309(0.51)\end{array}$ \\
\hline
\end{tabular}

Table 1 Linkage disequilibrium (LD) summary statistics of mean values of the Lewontin's normalized $D^{\prime}$ (Lewontin, 1964), the standardized $\chi^{\prime 2}$ (Yamazaki, 1977; Hedrick, 1987), and the proportion of marker pairs in significant $(P<0.05)$ LD in all linkage groups separately for syntenic and nonsyntenic markers

Values in parenthesis are standard errors for $D^{\prime}$ and $\chi^{\prime 2}$ and the percentages for $P<0.05 . n$ is the number of individuals analyzed. 
and data sets 3-7 comprised genotypes from the five largest families (Table 3), respectively. For the purpose of comparison to other studies, the measure $D^{\prime}$, which has been used widely in studies of non-human vertebrates (for example, Edwards and Dillon, 2004; Backström et al., 2006; Slate and Pemberton, 2007), was included in further analysis within and among the LGs. Plots of all pairwise comparisons among associations relative to genetic distances and the genetic diversity observed at the microsatellites, in addition to the frequency distribution of $D^{\prime}$ and cumulative frequency of Fisher exact $P$-values, were generated in Microsoft Excel. Analysis of variance was performed by SPSS release 15.0.1 statistical package and the Mantel test (10000 permutations) was implemented in $\mathrm{R}$ package.

\section{Results}

\section{Patterns of polymorphism and between-family genetic structure}

A total of 35557 autosomal and 661 Z-linked microsatellite genotypes in the sample were available for analysis. The average observed heterozygosity $\left(H_{\mathrm{O}}=0.56 \pm 0.003\right)$ for autosomal markers was significantly higher than those for gonosomal markers $\left(H_{\mathrm{O}}=0.27 \pm 0.01\right.$; Supplementary Figure S1). However, gonosomal markers were more differentiated (mean $F_{\mathrm{ST}}: 0.111 \pm 0.021$ ) than the autosomal markers (mean $F_{\mathrm{ST}}$ : $0.075 \pm 0.013$ ) among families (see Supplementary Figure S2). The genetic divergence among the families was high $\left(F_{\mathrm{ST}}=0.082,95 \% \mathrm{CI}=0.068-0.100\right)$, indicating substantial structure between the families. Among the individuals, the mean $F_{\text {IS }}$ across all the loci was $0.036( \pm 0.015)$ and deviated significantly $(P<0.05)$ from 0 .

\section{LD in syntenic markers in the pedigree}

LD was estimated for 951 syntenic marker pairs. Figure 1 and Table 1 show the distribution of $D^{\prime}$ values as a function of genetic distance in centimorgans. In general, $D^{\prime}$ decayed with distance varying between 0.025 and 0.913 (see Figures 1 and 2) and the 'half-length' of LD (measured as the distance at which mean $D^{\prime}$ falls to 0.5 ) is ca. $20 \mathrm{cM}$ (Figure 1). There was also extensive variability in the magnitude of $D^{\prime}$ at a given genetic distance reflecting wide local variations in the extent of LD across the LGs. Marker distance was significantly and negatively correlated with $D^{\prime}$ for markers spaced $<60 \mathrm{cM}$ apart (Mantel's test: $r=-0.22, n=579, P<0.001$ ), whereas markers separated by $>60 \mathrm{cM}$ showed no significant relationship with distance (Mantel's test: $r=-0.014$, $n=372, P>0.1)$. $D^{\prime}$ averaged $51.7 \%$ for marker pairs $<20 \mathrm{cM}$ apart, decaying relatively moderately to ca. $38 \%$ at distances of $60 \mathrm{cM}$, and then leveling slowly below $30 \%$ for markers separated by $100 \mathrm{cM}$ or more. However, significant $(P<0.05)$ and strong associations $\left(D^{\prime}>0.5\right)$ were observed also for many of the comparisons among loci separated by $>100 \mathrm{cM}$ (Table 1; Figure 1).

As compared with the LD measured by $D^{\prime}$, use of the $\chi^{\prime 2}$ metric resulted in a $50 \%$ reduction in LD estimates (Table 1). In particular, there seems to be much more variation in the value of $D^{\prime}$ at a given genetic distance than in the values of $\chi^{\prime 2}$ (Figure 1 ). In contrast, the mean estimate of LD within LGs decays more rapidly with increasing genetic distances $<60 \mathrm{cM}$ when measured with $\chi^{\prime 2}$ as compared to $D^{\prime}$ (Table 1 ; Figure 1 ).

LD was also further assessed within the LGs using the estimator $D^{\prime}$. Table 2 summarizes the frequency distribution of $D^{\prime}$ against genetic distance for the eight LGs. The highest frequency of observed values of $D^{\prime}$ was between 0.40 and 0.60 for all LGs except LG7, whereas the highest and lowest $D^{\prime}$ values differed between the LGs (Table 2). The mean $D^{\prime}$ varied between 0.461 and 0.726 , and the difference was highly significant among the LGs (one-way analysis of variance; $F_{7973}=6.46, P<0.0001$; Table 2).

There was a great deal of interchromosomal heterogeneity in the way LD varied as a function of physical distance between markers (Figure 2). Despite that pairwise LD decays significantly over ca. 5-20 cM in the five autosomal LGs (LG1, LG2, LG5, LG6, and LGZ), marker pairs on autosomal LG3, LG4, and LG7 showed elevated LD over distances that approach the total length of the LGs. At comparable distances (0-5, 5-10, 10-15, $15-20$, and $20-25 \mathrm{cM}), D^{\prime}$ values were systematically lower for the Z-linked marker pairs than for the autosomal marker pairs (data not shown; Figure 2). When logarithmic trend lines were fitted to the data of

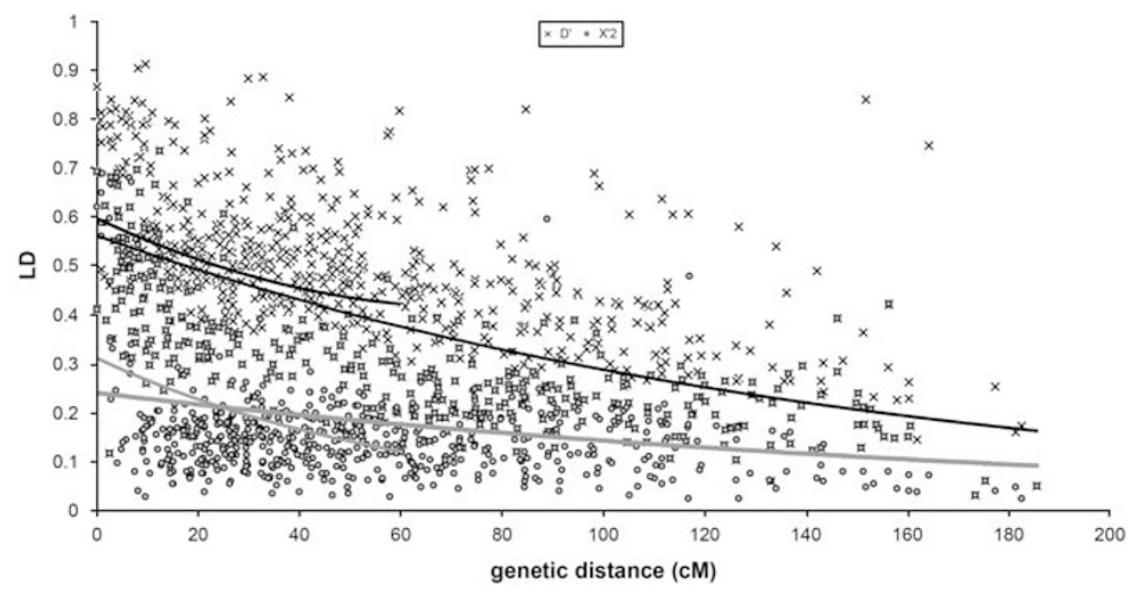

Figure 1 Linkage disequilibrium as measured by $D^{\prime}$ and $\chi^{\prime 2}$ as a function of genetic distances (cM) between autosomal syntenic markers for the overall distance (longer curves) and $>60 \mathrm{cM}$ (shorter curves). The dark and gray lines give exponential trend line fits of $D^{\prime}$ and $\chi^{\prime 2}$, respectively. 

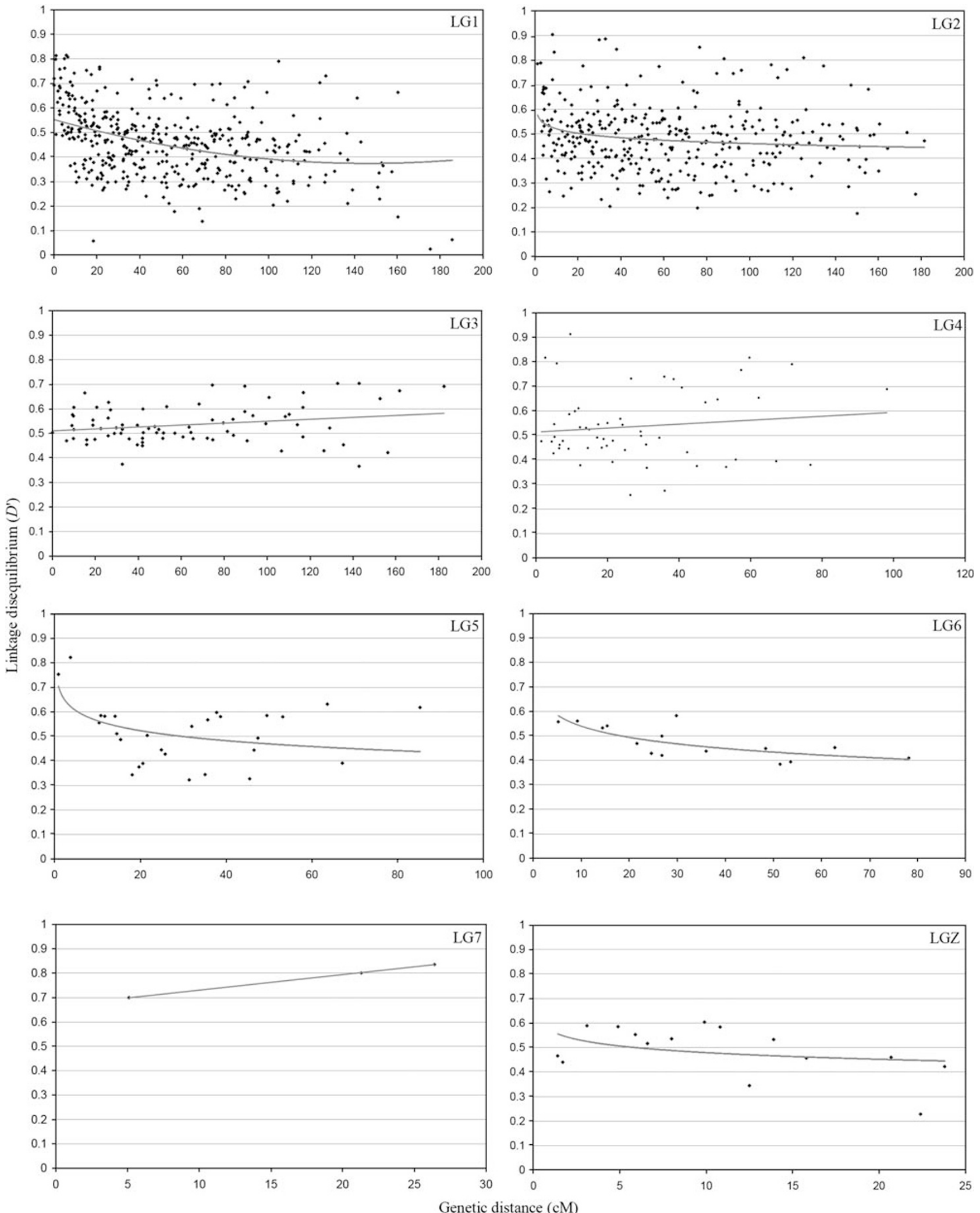

Figure 2 Linkage disequilibrium $\left(D^{\prime}\right)$ as a function of the genetic distances (cM) between marker pairs within the same LG. The second-order polynomial linear regression fits to the data of LG1, the logarithmic trend lines fit to the data of LG2, LG5, LG6, and LGZ; and the linear regression trend lines fit to the data of LG3, LG4, and LG7.

LG2, LG5, LG6, and LGZ, the coefficients of determination $\left(R^{2}\right)$ vary from 0.035 for LG2 to 0.556 for LG6, whereas the second-order polynomial linear regression fits best to the data of LG1 by the valid criterion of $R^{2}$ (Figure 2). All negative correlations between LD and genetic distances were significant $(P<0.05$ for LG1, LG2, 
Table 2 Distribution of $D^{\prime}$ against genetic distance within linkage groups

\begin{tabular}{|c|c|c|c|c|c|c|c|c|}
\hline \multirow[t]{2}{*}{ Linkage group (LG) } & \multirow[t]{2}{*}{ No. of loci } & \multirow[t]{2}{*}{ Length (cM) } & \multicolumn{6}{|c|}{$L D\left(D^{\prime}\right)$ bin } \\
\hline & & & $0.00-0.20$ & $0.20-0.40$ & $0.40-0.60$ & $0.60-0.80$ & $0.80-1.00$ & Mean $D^{\prime} \pm s . d$ \\
\hline LG1 & 29 & 185.6 & 0.002 & 0.279 & 0.556 & 0.153 & 0.007 & $0.476(0.122)$ \\
\hline LG2 & 27 & 181.5 & 0.006 & 0.262 & 0.595 & 0.120 & 0.022 & $0.480(0.132)$ \\
\hline LG3 & 13 & 182.6 & 0 & 0.026 & 0.769 & 0.192 & 0.013 & $0.534(0.076)$ \\
\hline LG4 & 11 & 98.2 & 0 & 0.164 & 0.564 & 0.218 & 0.055 & $0.535(0.144)$ \\
\hline LG5 & 8 & 85.2 & 0 & 0.214 & 0.607 & 0.143 & 0.036 & $0.526(0.124)$ \\
\hline LG6 & 6 & 78.2 & 0 & 0.200 & 0.800 & 0 & 0 & $0.461(0.071)$ \\
\hline LG7 & 3 & 26.4 & 0 & 0 & 0.333 & 0.667 & 0 & $0.726(0.161)$ \\
\hline LGZ & 6 & 23.8 & 0 & 0.125 & 0.75 & 0.125 & 0 & $0.501(0.113)$ \\
\hline
\end{tabular}

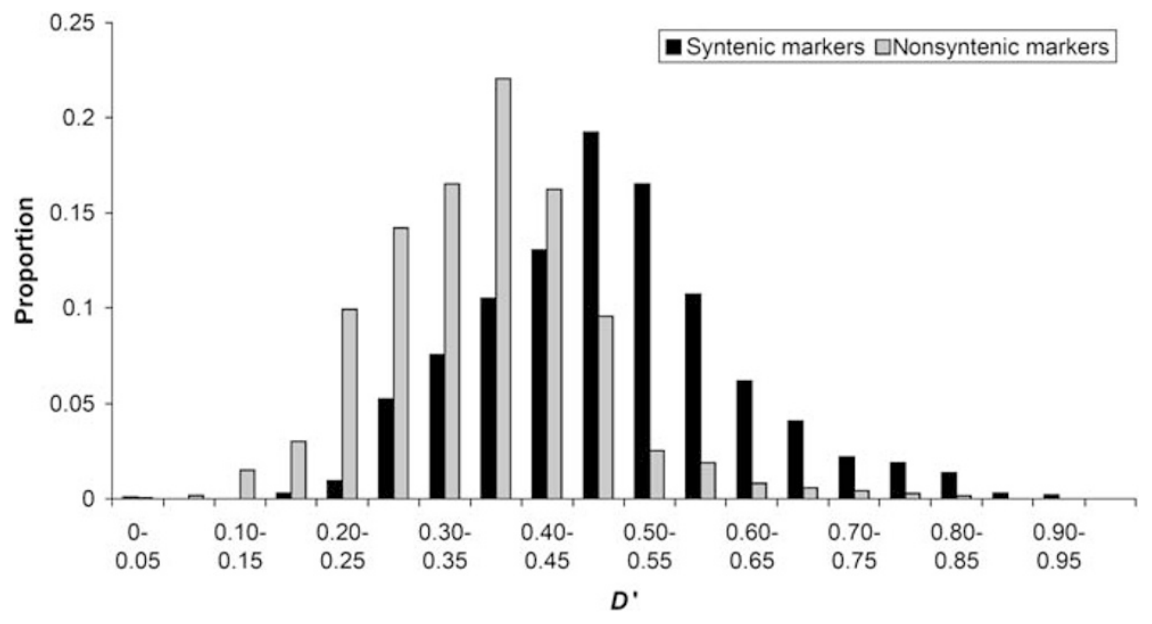

Figure 3 Frequency distributions of $D^{\prime}$ for syntenic and nonsyntenic marker pairs.

LG5, LG6, and LG7), but the positive correlations for LG3, LG4, and LG7 were nonsignificant $(P>0.05)$ by the Mantel permutation test. Moreover, the 'half-length' of LD also varied among the LGs (ca. 20 cM for LG1, LG2, and LG6, ca. $30 \mathrm{cM}$ for LG5, and ca. $5 \mathrm{cM}$ for LGZ; see Figure 2).

Whether LD in chromosomes is structured in blocklike' manner is of considerable interest as if it is so, it will simplify LD-mapping studies (Zhang et al., 2002). Again, the patterns of LD measured by the $D^{\prime}$ estimates are very different among the LGs (Supplementary Figure S3). For example, in LG3 and LG4, there are much less and isolated associations between the syntenic markers, whereas in LG1 and LG2 the associations are patchy, not block like. On the other hand, pairwise combinations of alleles at the syntenic loci show considerable difference in magnitude, significance, and patterns of interallelic disequilibrium among the LGs. It is clear that some regions within the LGs have extended LD whereas others do not (Supplementary Figure S3). In addition, $D^{\prime}$ values were not distributed uniformly across the nonsyntenic LD map. The results indicated again extensive LD within LGs (see Supplementary Figure S3). However, because of the large number of comparisons, none would be individually significant if Bonferroni corrections were applied.

Examination of the cumulative distribution of $P$-values for syntenic marker pairs showed a great proportion of syntenic marker pairs $(90.2 \%, 858 / 951)$ to be insignificant $(P<0.05)$ LD (Table 1; Supplementary Figure S4). Of the syntenic marker pairs separated by $20 \mathrm{cM}$ or less, 95\% $(211 / 222)$ were insignificant $(P<0.05)$ LD. However, substantial and significant $(P<0.05)$ LD were also observed among syntenic markers separated by $>60 \mathrm{cMs}$.

LD in nonsyntenic markers in the pedigree

Gametic LD determined for the 4309 nonsyntenic marker pairs and the distribution of $D^{\prime}$ values for nonsyntenic markers (0.091-0.824; Figure 3) overlapped strongly with that for syntenic markers (0.025-0.913; Figure 3), although nonsyntenic pairs did have a significantly lower mean $D^{\prime}(0.351 \pm 0.121)$ than nonsyntenic pairs (mean $D^{\prime}=0.439 \pm 0.151 ; t_{5258}=5.21, P<0.001$ ). Likewise, a significant lower mean $\chi^{\prime 2}$ was observed in pairs of nonsyntenic markers $(0.165 \pm 0.061)$ than that in syntenic pairs (mean $\chi^{\prime 2}=0.223 \pm 0.078 ; t_{5258}=4.61, P<0.001$ ).

We looked also for possible significant LD between nonsyntenic loci. Examination of the cumulative frequency distribution of $P$-values between nonsyntenic loci indicates that significant $(P<0.05)$ LD occurs in $82.8 \%(3567 / 4309)$ of the marker pairs. In addition, as shown in Table 1, the average $D^{\prime}$ value measured between nonsyntenic marker pairs was 0.354 , therefore quite similar to the $D^{\prime}$ values $(0.356)$ found for distant $(>60 \mathrm{cM})$ though syntenic loci. Hence, the data from the Siberian jay pedigree support the presence of significant long-range LD among nonsyntenic markers.

LD in the founders and the families

LD between syntenic and nonsyntenic markers was also examined in the founders as well as within the 
Table 3 Linkage disequilibrium (LD) summary statistics of mean values of the Lewontin's normalized $D^{\prime}$ (Lewontin, 1964) against intervals of genetic distances within the five large families

\begin{tabular}{|c|c|c|c|c|c|c|c|}
\hline \multirow[t]{2}{*}{ Data set } & \multirow[t]{2}{*}{$\mathrm{n}$} & \multicolumn{5}{|c|}{ Syntenic } & \multirow[t]{2}{*}{ Nonsyntenic } \\
\hline & & $0-20 c M$ & $20-40 c M$ & $40-60 c M$ & $60-100 c M$ & 100-190 cM & \\
\hline 3 (Family A) & 70 & $0.554(0.166)$ & $0.492(0.151)$ & $0.487(0.139)$ & $0.421(0.131)$ & $0.356(0.109)$ & $0.382(0.117)$ \\
\hline 4 (Family B) & 65 & $0.532(0.159)$ & $0.499(0.148)$ & $0.491(0.141)$ & $0.436(0.135)$ & $0.371(0.114)$ & $0.387(0.119)$ \\
\hline 5 (Family C) & 69 & $0.523(0.149)$ & $0.486(0.147)$ & $0.478(0.136)$ & $0.409(0.127)$ & $0.366(0.107)$ & $0.369(0.115)$ \\
\hline 6 (Family D) & 72 & $0.534(0.158)$ & $0.489(0.149)$ & $0.481(0.137)$ & $0.411(0.129)$ & $0.373(0.112)$ & $0.376(0.109)$ \\
\hline 7 (Family E) & 73 & $0.519(0.138)$ & $0.486(0.131)$ & $0.475(0.128)$ & $0.391(0.119)$ & $0.325(0.111)$ & $0.343(0.118)$ \\
\hline
\end{tabular}

$n$ is the number of individuals analyzed.

five largest families (Tables 1 and 3). In general, LD between syntenic markers was lower in data set 2 (the founders) than in data set 1 (the whole Suupohja pedigree) either measured by $D^{\prime}\left(F_{1,951}=9.31, P<0.05\right)$ or by $\chi^{\prime 2}\left(F_{1,951}=4.67, P<0.05\right)$. A larger proportion of marker pairs in significant LD were also observed in syntenic markers $(3567 / 4309,0.83)$ than in nonsyntenic markers (2213/4309, 0.51; Table 1). However, despite statistically significant differences in levels of LD in pedigree and founder data, the actual differences in LD levels were slight (Table 1). This together with the similar decay of LD with physical distance in the two data sets suggests that the extent and patterns of LD in the pedigree data reflect that in the whole population in general.

Table 3 shows the mean $D^{\prime}$ against different intervals of genetic distance for syntenic marker pairs as well as for nonsyntenic marker pairs in each family. The results indicate systematically higher LD for the withinfamily data than for the whole pedigree data both for syntenic and nonsyntenic marker pairs (Table 3). However, the actual numerical differences in the levels of LD between family and pedigree data are slight (see Tables 1 and 3). Furthermore, a similar rate of decay with genetic distance occurs between each family and the whole pedigree in terms of the mean $D^{\prime}$ (Tables 1 and 3).

\section{Discussion}

This work is so far the most comprehensive study of LD in a wild bird population. We discovered very high levels of LD across the genome of Siberian jays. LD extended for tens of centimorgans within LGs and occurred also among nonsyntenic markers suggesting extensive LD in the genome of the Siberian jay. However, LD did decay with genetic distance across the genome, and there was marked heterogeneity in the extent of LD among LGs. Nevertheless, as compared with other species for which information about the levels and extent of LD is available (for example, Edwards and Dillon, 2004; Backström et al., 2006; Slate and Pemberton, 2007; Balakrishnan and Edwards, 2009), the patterns and levels of LD uncovered in this study are clearly exceptional. Although part of the explanation may reside on the fact that our data consisted to a certain extent of closely related individuals from large pedigreed families, the fact that the extent and patterns of LD were very similar irrespectively whether whole pedigree, founder, or family data were used suggests that the results reflect the 'true' situation in this population.

\section{LD among syntenic markers}

Considerable amounts of LD between loci were detected by both the $D^{\prime}$ and $\chi^{\prime 2}$-statistics. The large difference in the degree of LD indicated by these two measures was not unexpected (see the review by Slatkin, 2008) and the amount of LD decayed with increasing distance between markers for both metrics.

LD was found to occur for marker pairs separated by long genetic distances. The 'half-length' of LD that was chosen in this study was the same as previously used in LD studies of human (Reich et al., 2001), cattle (Farnir et al., 2000), dog (Sutter et al., 2004), chicken (Andreescu et al., 2007), and collared flycatcher (Backström et al., 2006) populations. However, the extensive LD in the Siberian jay extended over much longer distances than that observed in the above-mentioned species. Assuming a similar mean recombination rate as in another passerine bird-the zebra finch $\left(3.18 \mathrm{cM} \mathrm{Mb}^{-1}\right.$; Stapley et al., 2008) — the 'half-length' of LD in Siberian jays is ca. $6.28 \mathrm{Mb}\left(=20(\mathrm{cM}) / 3.18\left(\mathrm{cM} \mathrm{Mb}^{-1}\right)\right)$ in physical distance. The 'half-length' of LD varied from 5 to $10 \mathrm{cM}$ in the domestic livestock populations, whereas appreciable LD were detected only among markers separated by $1 \mathrm{cM}$ (Andreescu et al., 2007) or up to $5 \mathrm{cM}$ (Heifetz et al., 2005) in chicken. However, studies of wild bird populations have produced heterogenous results to this effect. Edwards and Dillon (2004) found low levels of LD at small genomic scales in autosomes of red-winged blackbird Agelaius phoeniceus, whereas Backström et al. (2006) found relatively higher levels of LD with the half-length of less than $1 \mathrm{cM}$ on the Z-chromosome of the collared flycatcher. Even lower levels of LD-even over as short distances as $300 \mathrm{bp}$-were detected in the zebra finch Taeniopygia guttata populations (Balakrishnan and Edwards, 2009). Nevertheless, these latter results were based on a low number of spaced markers. In the studies of other wild vertebrate populations, relatively high levels of LD extended over several tens of centimorgans in the global pattern of red deer (Slate and Pemberton, 2007), which are comparable with that found in the Siberian jay.

High levels of LD can be built up in small and subdivided populations because small effective size and admixture between genetically divergent lineages are expected to increase levels of LD (for example, Peltonen et al., 2000). These factors may interactively influence the observed high levels of LD in the Siberian jays, which have undergone long-term isolation (Lillandt et al., 2001, 2003) and within which significant inbreeding (Alho et al., 2009; $F_{\mathrm{IS}}=0.036, P<0.05$, this study) and population substructure $\left(F_{\mathrm{ST}}=0.082, P<0.05\right)$ have been found. 
Although high levels of LD was observed in this study, direct comparisons with other studies should be done with caution because of the differences in measurement metrics, genetic map units, mating system, the targeted genomic region studied (for example, the MHC region studied in red-winged blackbirds (Edwards and Dillon, 2004) - a region that is know to have extremely high recombination rates in mammals (for example, Dumont and Payseur, 2008) and birds (for example, Kaufman and Salomonsen, 1997)) and the molecular markers used etc (see for example, Slate and Pemberton, 2007; Meadows et al., 2008). Another potential caveat in the comparisons concerns the variance in relatedness among individuals in different data sets. For instance, as in case of the family data used here, recombination has not yet had much time to break up associations between loci and it is therefore very hard to compare the extent and level of LD observed here with that in other populations. As further indicated by the results from the different data sets, LD was significantly greater in the pedigree (data set 1) than the founders (data set 2), which were less related to each other (Table 1). In data set 1 , the associations between markers, particularly the nonrecombinant ones, have been represented repeatedly among the descendants of a small number of highly successful individuals such as some of the founders (see Slate and Pemberton, 2007). However, high levels of LD were still observed in the founders, indicating that the inference is likely to apply to the whole population too. As the pedigree is part of a larger data set, some of the 'founders' might be still related if the pedigree of the whole data set could be resolved. In addition, the behavior of LD-as measured by the $D^{\prime}$ metric-was found to differ between the families being systematically higher within the families than in the whole pedigree. This could be explained by the closer relatedness between the family samples (c.f. most of them are full-sibs) than that between samples from the whole pedigree, although the effect of population structure on LD may have been reduced. The persistence of LD over long distances within the families is therefore not surprising. A small effective population size and closely related individuals may lie behind the high LD observed in this study.

\section{Variation in LD among LGs}

Our results showed considerable variation in LD not only between Z-chromosomal and automsomal LGs, but also among different autosomal LGs (Figure 2). Similar interchromosomal heterogeneity in LD among syntenic marker pairs has been observed also in rice (Mather et al., 2007), pigs (Nsengimana et al., 2004), and red deer (Slate and Pemberton, 2007). Differences in local recombination rates may account for this heterogeneity (see Yu et al., 2001). However, the effect should be slight as the local recombination rate always varies at shorter scales than what can be detected by markers separated by a few kilobases (see for example, Pritchard and Przeworski, 2001), rather than several and even tens of centimorgans as in this study. Another potential explanation could be the 'Bulmer effect' describing a higher LD between genomic regions harboring QTL undergoing selection (Bulmer, 1971). Despite the fact that $D^{\prime}$ is assumed to be a frequency-independent measure (Hedrick, 1987), the significant linkage-group effect could still be attributable to heterogeneity in number of marker pairs in LGs (Slate and Pemberton, 2007). Likewise, the heterogenous karyotype and chromosomal structure of birds with few macrochromosomes and many microchromosomes (Balakrishnan and Edwards, 2009) could at least partly explain our finding.

When examining the relationship between the LD and genetic distance, a progression of decreasing association, measured with the $D^{\prime}$ metric, was not always observed in the different LGs (Figure 2). This observation parallels that described in humans (Jeffreys et al., 2001), barley (Caldwell et al., 2006), and zebra finch (Balakrishnan and Edwards, 2009). One plausible explanation for the tendency of LD to increase with physical distance is the presence of contrasting locus histories within the same LG. The relationship between LD and genetic distance might also be shaped by gene conversion. For example, for closely linked markers, LD may also be broken up by gene conversion (see Pritchard and Przeworski, 2001). Indeed, gene conversion has been suggested to be quite frequent in the Siberian jay, although the precise details are unknown (Jaari et al., 2009). Another explanation for difference in rate of decay in LD between LGs is that the precision of linkage map distances differs between markers and LGs because of number of markers used, marker variability, and genotyping errors (see Jaari et al., 2009).

\section{LD on the Z-chromosome}

The levels of polymorphism and LD for microsatellites were found to be significantly lower in Z-chromosome than in autosomes at comparable genetic distances by pairwise comparisons (Figures 1 and 3; Table 1). However, an increased level of LD among Z-chromosomal markers was observed only among males ( $\mathrm{Li}$ and Merilä, unpublished data). In the combined sample of both sexes, the reduced LD on the $\mathrm{Z}$ is in marked contrast to typical observations of higher LD on the $X$ chromosome in mammals as in the case of humans (for example, Altshuler et al., 2005), cattle (for example, Sandor et al., 2006), and wild mice (for example, Laurie et al., 2007). However, the findings would be compatible with theoretical expectations for differential selection on sex-linked loci under male and female heterogamy (Ellegren, 2009), as well as with the demonstration that the Z-chromosome is likely to be particularly sensitive to sexual selection in female heterogamy systems as compared to the $X$ chromosome in $X X / X Y$ systems (see Reeve and Pfennig, 2003; Backström et al., 2006). Another potential source for the reduction in LD on the Z-chromosome may concern the three pseudoautosomal markers (Jaari et al., 2009), which constitute a large part of the Siberian jay LGZ. Recombination rates between markers in pseudoautosomal region are extremely high as compared with genomic averages because of obligate chiasma formation in this short region in every meiosis in the heterogamtic sex as supported by mammalian studies (for example, Huang et al., 2005). However, earlier studies in mammals included only or mostly X-specific markers, which in general show higher levels of LD than the pseudoautosomal markers (see Marshall et al., 2004; Sandor et al., 2006). 
LD among nonsyntenic markers and prospects for LD mapping

The high level of LD is of great interest for future mapping studies of the Siberian jays, as it means that large quantitative trait regions rather than QTLs can be detected. Another striking feature of our results was the high proportion of nonsyntenic marker pairs in significant LD (Supplementary Figure S4). Earlier studies of LD have observed a mean nonsyntenic LD of $10-40 \%$ in domestic livestock (Farnir et al., 2000; McRae et al., 2002). However, $69-77 \%$ of nonsyntenic marker pairs were found to be in LD in a natural population of red deer (Slate and Pemberton, 2007), in which the pattern was ascribed to a recent admixture event. In this study, a potential explanation for the strong background LD is in the significant intrapopulation genetic structuring and the relatedness (c.f. pedigree samples) among individuals. Other potential explanations include small effective population size (c.f. Nsengimana et al., 2004) of the study population $\left(N_{\mathrm{e}}=170\right.$; Henna Fabritius, unpublished MSc thesis), as well as the sampling of related individuals in the pedigree. Whatever the explanation, the common occurrence of LD among nonsyntenic loci evokes serious concerns about the generation of type I errors when using LD mapping as the only means to locate genes underlying complex traits in the population (Farnir et al., 2000). Therefore, a new strategy of joint linkage and LD mapping in natural populations (Wu and Zeng, 2001), which has the power to simultaneously capture the information about the linkage of the markers (as measured by recombination rate) and the degree of historical LD, would be preferred in a situation like this. Likewise, the considerable LD over long distances reduces precision in association-mapping context (Pritchard and Przeworski, 2001). A potential solution to this problem is to map QTLs in several populations simultaneously as the successful validation of QTLs depends on the sign of the association in different populations rather than on the strength of the initial association or its size of effect (Barendse et al., 2007).

\section{Molecular markers and LD metrics-some additional considerations}

Although biallelic SNP markers are likely to predominate in LD-mapping studies of human populations, multiallelic microsatellites will continue to be useful in LD-mapping studies of livestock and wild vertebrates (for example, Farnir et al., 2000; Slate and Pemberton, 2007; Lipkin et al., 2009). The values obtained here for both $D^{\prime}$ and $\chi^{\prime 2}$ at the $<20 \mathrm{cM}$ range are much greater than those reported for two other wild passerines (the collared flycatcher Ficedula albicollis, Backström et al., 2006; the zebra finch T. guttata, Balakrishnan and Edwards, 2009) for biallelic SNP markers, using $D^{\prime}$ and the comparable $r^{2}$ measure. Thus, LD between multiallelic markers may indeed be greater and extend over longer distances than LD among biallelic SNP markers, which was also found in the LD studies in humans (Pritchard and Przeworski, 2001; Varilo et al., 2003) and cattle (Khatkar et al., 2006a,b). In addition, the relatively high rate of mutation in microsatellite markers makes possible the assessment of recently created LD
(Mountain et al., 2002). Thus, microsatellite markers will continue to be useful and informative for studies of LD in the wild.

Currently there is no consensus with respect to the relative merits of the different measures (for example, $D^{\prime}, \chi^{\prime 2}$, and $r^{2}$ ) of LD (but see Hudson, 2001). The metrics may have different context-dependent strengths. Slate and Pemberton (2007) recommended reporting both $D^{\prime}$ and $\chi^{\prime 2}$ in the future studies to allow comparison of LD statistics across studies. As suggested, we calculated both $D^{\prime}$ and $\chi^{\prime 2}$ metrics and chose to use $D^{\prime}$ in the further detailed analyses because the good biological and statistical properties of $\chi^{\prime 2}$ may be outweighed by its frequency dependence and variable rate of decay (for example, Hedrick, 1987; Nsengimana et al., 2004). Furthermore, use of $D^{\prime}$ facilitates the comparison of our results with those of wild vertebrate populations (for example, collared flycatcher, Backström et al., 2006; red deer, Slate and Pemberton, 2007; and zebra finch, Balakrishnan and Edwards, 2009). In particular, when comparing samples with different allelic frequencies or different pairs of loci a frequency-independent measure such as $D^{\prime}$ is quite important (Hedrick, 1987). Moreover, $D^{\prime}$ is the measure of choice to assess recombination patterns as LD blocks have often been defined on the basis of $D^{\prime}$ (Chen et al., 2006).

\section{Conclusions}

Knowledge about LD is important in the context of performing genome-wide association analysis and understanding strength and patterns of genomic selection (for example, Meadows et al., 2008). The high levels of LD observed here suggest good prospects for association studies in this population, although ways to minimize the generation of false-positive results would need to be adopted. The patterns observed in this study also provide insights into the role of genomic selection in shaping, for example, patterns of genome variability and LD on the avian Z-chromosome. Furthermore, the findings presented here, together with those of a few other studies (Edwards and Dillon, 2004; Backström et al., 2006; Balakrishnan and Edwards, 2009), indicate that wild birds could provide useful models for identifying genes underlying variation in complex traits of ecological and evolutionary interest.

\section{Conflict of interest}

The authors declare no conflict of interest.

\section{Acknowledgements}

This work was supported by grants from the Academy of Finland, University of Helsinki, and Ministry of Education. We thank Bo-Göran Lillandt for collection of the primary data. Thanks are also due to all individuals and funding bodies, which have-directly or indirectlycontributed to collection of the data. We are grateful to Sonja Jaari, Kaisa Välimäki for her help in the molecular data preparation, and Marika Karjalainen for technical assistance in laboratory. Niclas Backström, John Loehr, and four anonymous referees kindly and constructively commented on earlier versions of this paper. 


\section{References}

Alho JS, Lillandt BG, Jaari S, Merilä J (2009). Multilocus heterozygosity and inbreeding in the Siberian jay. Conserv Genet 10: 605-609.

Altshuler D, Brooks LD, Chakravarti A, Collins FS, Daly MJ, Donnelly P (2005). A haplotype map of the human genome. Nature 437: 1299-1320.

Andreescu C, Avendano S, Brown SR, Hassen A, Lamont SJ, Dekkers CM (2007). Linkage disequilibrium in related breeding lines of chicken. Genetics 177: 2161-2169.

Ardlie KG, Kruglyak L, Seiestad M (2002). Patterns of linkage disequilibrium in the human genome. Nat Rev Genet 3: 299-309.

Backström N, Qvarnström A, Gustafsson L, Ellegren H (2006). Levels of linkage disequilibrium in a wild bird population. Biol Lett 2: 435-438.

Balakrishnan C, Edwards S (2009). Nucleotide variation, linkage disequilibrium and founder-facilitated speciation in wild populations of the zebra finch (Taeniopygia guttata). Genetics 181: 645-660.

Barendse W, Reverter A, Bunch RJ, Harrison BE, Barris W, Thomas MB (2007). A valiated whole genome association study of efficient food conversion in cattle. Genetics $\mathbf{1 7 6}$ 1893-1905.

Bulmer MG (1971). The effect of selection on genetic variability. Am Nat 105: 201-211.

Caldwell KS, Russell J, Langridge P, Powell W (2006). Extreme population-dependent linkage disequilibrium detected in an inbreeding plant species, Hordeum vulgare. Genetics 172: $557-567$.

Chen Y, Lin CH, Sabatti C (2006). Volume measures for linkage disequilibrium. BMC Genet 7: 54.

De La Vega FM, Isaac H, Collins A, Scafe CR, Halldórsson BV, $\mathrm{Su} X$ et al. (2005). The linkage disequilibrium maps of three human chromosomes across four populations reflect their demographic history and a common underlying recombination pattern. Genome Res 15: 454-462.

Dumont BL, Payseur BA (2008). Evolution of the genomic rate of recombination in mammals. Evolution 62: 276-294.

Edwards SV, Dillon M (2004). Hitchhiking and recombination in birds: evidence from MHC-linked and unlinked loci in red-winged blackbirds (Agelaius phoeniceus). Genet Res 84: 175-192.

Ekman J, Baglione V, Eggers S, Griesser M (2001). Delayed dispersal: living under the region of nepotistic parents. Auk 118: 1-10.

Ekman J, Sklepkovych B, Tegelström H (1994). Offspring retention in the Siberian jay (Perisoreus infaustus): the prolonged brood care hypothesis. Behav Ecol 5: 245-253.

Ellegren H (2009). Genomic evidence for a large-Z effect. Proc $R$ Soc B 276: 361-366.

Ellegren H, Sheldon BC (2008). Genetic basis of fitness differences in natural populations. Nature 452: 169-175.

Farnir F, Coppieters W, Arranz JJ, Berzi P, Cambisano N, Grisart B et al. (2000). Extensive genome-wide linkage disequilibrium in cattle. Genome Res 10: 220-227.

Flint-Garcia SA, Thornsberry JM, Bucker ES (2003). Structure of linkage disequlibrium in plants. Annu Rev Plant Biol 54: 357-374.

Gaunt TR, Rodriguez S, Zapata C, Day INM (2006). MIDAS: software for analysis and visulisation of interallelic disequlibrium between multiallelic markers. BMC Genomics 7: 227.

Goudet J (2002). FSTAT, a program to estimate and test gene diversities and fixation indices (version 2.9.3.2). Available from http://www.unil.ch/izea/softwares/fstat.html, updated from Goudet (1995).

Gracey A, Cossins A (2003). Application of microarray technology in environmental and comparative physiology. Annu Rev Physiol 65: 231-259.

Gratten J, Wilson AJ, McRae AF, Beraldi D, Visscher PM, Pemberton JM et al. (2008). A localized negative genetic correlation constrains microevolution of coat color in wild sheep. Science 319: 318-320.

Green P, Falls K, Crooks S (1990). Documentation for CRI-MAP. Washington University School of Medicine: St Louis.

Hedrick P (1987). Gametic disequilibrium measures: proceed with caution. Genetics 117: 331-341.

Heifetz EM, Fulton JE, O'Sullivan N, Zhao H, Dekkers JCM, Soller M (2005). Extent and consistency across generations of linkage disequilibrium in commercial layer chicken breeding populations. Genetics 171: 1173-1181.

Hill WG, Robertson A (1968). Linkage disequilibrium in finite populations. Theor Appl Genet 38: 226-231.

Huang SW, Friedman R, Yu N, Yu A, Li WH (2005). How strong is the mutagenicity of recombination in mammals? Mol Biol Evol 22: 426-431.

Hudson RR (2001). Two-locus sampling distributions and their application. Genetics 159: 1805-1817.

Jaari S, Li MH, Merilä J (2009). A first-generation microsatellitebased genetic linkage map of the Siberian jay (Perisoreus infaustus): insights into avian genome evolution. BMC Genomics 10: 1.

Jeffreys U, Kauppi L, Neumann R (2001). Intensely punctuate meiotic recombination in the class II region of the major histocompatibility complex. Nat Genet 29: 217-222.

Kaufman J, Salomonsen J (1997). The 'Minimal Essential MHC' revisited: both peptide binding and cell surface expression level of MHC molecules are polymorphisms selected by pathogens in chickens. Hereditas 127: 67-73.

Kemppainen J (2002). Kuukkelihavainnot Etelä-Suomessa 1990-2001. Hippiäinen 32: 23-28.

Khatkar MS, Collins A, Cavanagh JAL, Hawken RJ, Hobbs M, Zenger KR et al. (2006a). A first-generation metric linkage disequilibrium map of bovine chromosome 6. Genetics $\mathbf{1 7 4}$ 79-85.

Khatkar MS, Thomson PC, Tammen I, Cavanagh JAL, Nicholas FW, Raadsma H (2006b). Linkage disequilibrium on chromosome 6 in Australian Holstein-Friesian cattle. Genet Sel Evol 38: 463-477.

Kolkman JM, Berry ST, Leon AJ, Slabaugh MB, Tang S, Gao W et al. (2007). Single nucleotide polymorphisms and linkage disequilibrium in sunflower. Genetics 177: 457-468.

Laurie CC, Nickerson DA, Anderson AD, Weir BS, Livingston RJ, Dean MD et al. (2007). Linkage disequilibrium in wild mice. PLoS Genet 8: e144.

Lewontin RC (1964). The interaction of selection and linkage. I. General considerations: heterotic models. Genetics 49: 49-67.

Lillandt BG, Bensch S, von Schantz T (2001). Parentage determination in kin-structured populations: microsatellite analyses in the Siberian jay Perisoreus infaustus during a 25-year population study. Avian Sci 1: 3-14.

Lillandt BG, Bensch S, von Schantz T (2003). Family structure in the Siberian jay as revealed by microsatellite analyses. Condor 105: 505-514.

Lipkin E, Straus K, Stein RT, Bagnato A, Schiavini F, Fontanesi L et al. (2009). Extensive long range and non-syntenic linkage disequilibrium in livestock populations: deconstruction of a conundrum. Genetics 181: 691-699.

Marshall AR, Knudsen KL, Allendorf FW (2004). Linkage disequilibrium between the pseudoautosomal PEPB-1 locus and the sex-determining region of chinook salmon. Heredity 93: 85-97.

Mather KA, Caicedo AL, Polato NR, Olsen KM, McCounch S, Purugganan MD (2007). The extent of linkage disequilibrium in rice (Oryza sativa L.). Genetics 177: 2223-2232.

McRae AF, McEwan JC, Dodds KG, Wilson T, Crawford AM, Slate J (2002). Linkage disequilibrium in domestic sheep. Genetics 160: 1113-1122.

McRae AF, Pemberton JM, Visscher PM (2005). Modelling linkage disequilibrium in natural populations: the example of the Soay sheep population of St Kilda, Scotland. Genetics 171: 251-258. 
Meadows JRS, Chan EKF, Kijas JW (2008). Linkage disequilibrium compared between five populations of domestic sheep. BMC Genet 9: 61.

Mohlke KL, Lange EM, Valle TT, Ghosh S, Magnuson VL et al. (2001). Linkage disequilibrium between microsatellite markers extends beyond $1 \mathrm{cM}$ on chromosome 20 in Finns. Genome Res 11: 1221-1226.

Mountain JL, Knight A, Jobin M, Gignoux C, Miller A, Lin AA et al. (2002). SNPSTRs: empirically derived, rapidly typed, autosomal haplotypes for inference of population history and mutational processes. Genome Res 12: 1766-1772.

Nordborg M, Borevitz JO, Bergelson J, Berry CC, Chory J, Hagenblad J et al. (2002). The extent of linkage disequilibrium in Arabidopsis thaliana. Nat Genet 30: 190-193.

Nsengimana J, Baret P, Halley C, Visscher PM (2004). Linkage disequilibrium in the domesticated pig. Genetics 166: 1395-1404.

Park SDE (2001). Trypanotolerance in West African cattle and the population genetic effects of selection. PhD thesis, University of Dublin, Dublin (2001).

Peltonen L, Palotie A, Lange K (2000). Use of population isolates for mapping complex traits. Nat Rev Genet 1: 182-190.

Pritchard JK, Przeworski M (2001). Linkage disequilibrium in humans: models and data. Am J Hum Genet 69: 1-14.

Rafalski A, Morgante M (2004). Corn and humans: recombination and linkage disequilibrium in two genomes of similar size. Trends Genet 20: 103-111.

Reeve HK, Pfennig DW (2003). Genetic basis for showy males: are some genetic systems especially conductive to sexual selection? Proc Natl Acad Sci USA 100: 1089-1094.

Reich D, Cargill M, Bolk S, Ireland J, Sabeti PC, Richter DJ et al. (2001). Linkage disequilibrium in the human genome. Nature 411: 199-204.

Remington DL, Thornsberry JM, Matsuoka Y, Wilson LM, Whitt SR, Doebley J et al. (2001). Structure of linkage disequilibrium and phenotypic association in the maize genome. Proc Natl Acad Sci USA 98: 11479-11484.

Sandor C, Farnir F, Hansoul S, Coppieters W, Meuwissen T, Georges M (2006). Linkage disequilibrium on the bovine $\mathrm{X}$ chromosome: characterization and use in quantitative trait locus mapping. Genetics 173: 1777-1786.

Schneider S, Roessli D, Excoffier L (2000). Arlequin Version 2.000: A Software for Genetic Data Analysis. Genetics and Biometry Laboratory, University of Geneva: Geneva.
Slate J, Pemberton JM (2007). Admixture and patterns of linkage disequilibrium in a free-living vertebrate population. $J$ Evol Biol 20: 1415-1427.

Slatkin M (1994). Linkage disequilibrium in growing and stable population. Genetics 137: 331-336.

Slatkin M (2008). Linkage disequilibrium-understanding the evolutionary past and mapping the medical future. Nat Rev Genet 9: 477-485.

Stapley J, Birkhead TR, Burke T, Slate J (2008). A linkage map of the zebra finch Taeniopygia guttata provides new insights into avian genome evolution. Genetics 179: 651-667.

Stephens JC, Schneider JA, Tanguay DA, Choi J, Acharya T, Stanley SE et al. (2001). Haplotype variation and linkage disequilibrium in 313 human genes. Science 293: 489-493.

Sutter NB, Eberle MA, Parker HG, Pullar BJ, Kirkness EF, Kruglyak L et al. (2004). Extensive and breed-specific linkage disequilibrium in Canis familiaris. Genome Res 14: 2388-2396.

Varilo T, Paunio T, Parker A, Perola M, Meyer J, Terwilliger JD et al. (2003). The interval of linkage disequilibrium (LD) detected with microsatellite and SNP markers in chromosomes of Finnish populations with different histories. Hum Mol Genet 12: 51-59.

Weir BS, Cockerham CC (1984). Estimating F-statistics for the analysis of population structure. Evolution 38: 1358-1370.

Wilson DJ, Gabriel E, Leatherbarrow AJ, Cheesbrough J, Gee S, Bolton E et al. (2008). Tracing the source of campylobacteriosis. PloS Genet 4: e1000203.

Wright S (1951). The genetical structure of populations. Ann Eugen 15: 323-354.

Wu RL, Zeng ZB (2001). Joint linkage and linkage disequilibrium mapping in natural populations. Genetics 157: 899-901.

Yamazaki T (1977). The effects of overdominance on linkage in a multilocus system. Genetics 86: 227-236.

Yu N, Zhao Z, Fu YX, Sambuughin N, Ramsay M, Jenkins T et al. (2001). Global patterns of human DNA sequence variation in a 10-kb region on chromosome 1. Mol Biol Evol 18: $214-222$

Zhang K, Deng M, Cheng T, Waterman MS, Sun F (2002). A dynamic programming algorithm for haplotype block partitioning. Proc Natl Acad Sci USA 99: 7335-7339.

Zhao H, Nettleton D, Soller M, Deckers JCM (2005). Evaluation of linkage disequilibrium measures between multi-allelic markers as predictors of linkage disequilibrium between markesr and QTL. Genet Res 86: 77-87.

Supplementary Information accompanies the paper on Heredity website (http://www.nature.com/hdy) 